TITLE:

\title{
Pressure induced phase transformation of Ba8Ga16Ge30 clathrate studied by $x$-ray diffraction and Raman spectroscopy
}

\section{AUTHOR(S):}

Kume, Tetsuji; Ohno, Satoshi; Sasaki, Shigeo; Shimizu, Hiroyasu; Ohishi, Yasuo; Okamoto, Norihiko L.; Kishida, Kyosuke; Tanaka, Katsushi; Inui, Haruyuki

\section{CITATION:}

Kume, Tetsuji ...[et al]. Pressure induced phase transformation of Ba8Ga16Ge30 clathrate studied by x-ray diffraction and Raman spectroscopy. JOURNAL OF APPLIED PHYSICS 2010, 107(1): 013517.

\section{ISSUE DATE:}

2010-01

URL:

http://hdl.handle.net/2433/147187

\section{RIGHT:}

Copyright 2010 American Institute of Physics. This article may be downloaded for personal use only. Any other use requires prior permission of the author and the American Institute of Physics. The following article appeared in JOURNAL OF APPLIED PHYSICS107, 013517 (2010) and may be found at

http://link.aip.org/link/APPLAB/v96/i21/p213515_s1 


\title{
Pressure induced phase transformation of $\mathrm{Ba}_{8} \mathrm{Ga}_{16} \mathrm{Ge}_{30}$ clathrate studied by $x$-ray diffraction and Raman spectroscopy
}

\author{
Tetsuji Kume, ${ }^{1, a)}$ Satoshi Ohno, ${ }^{1}$ Shigeo Sasaki, ${ }^{1,2}$ Hiroyasu Shimizu, ${ }^{1,2}$ Yasuo Ohishi, ${ }^{3}$ \\ Norihiko L. Okamoto, ${ }^{4}$ Kyosuke Kishida, ${ }^{4}$ Katsushi Tanaka, ${ }^{4}$ and Haruyuki Inui ${ }^{4}$ \\ ${ }^{1}$ Department of Materials Science and Technology, Gifu University, 1-1 Yanagido, Gifu 501-1193, Japan \\ ${ }^{2}$ Environmental and Renewable Energy Systems, Graduate School of Engineering, Gifu University, \\ 1-1 Yanagido, Gifu 501-1193, Japan \\ ${ }^{3}$ Japan Synchrotron Radiation Research Institute, Mikazuki-cho, Hyogo 679-5198, Japan \\ ${ }^{4}$ Department of Materials Science and Engineering, Kyoto University, Kyoto 606- 8501, Japan
}

(Received 21 August 2009; accepted 24 November 2009; published online 13 January 2010)

\begin{abstract}
The phase transition and the vibrational properties of $\mathrm{Ba}_{8} \mathrm{Ga}_{16} \mathrm{Ge}_{30}$ have been investigated at high pressures up to $40 \mathrm{GPa}$ at room temperature. The combined study of the high-pressure Raman and synchrotron powder x-ray diffraction (XRD) experiments revealed the occurrence of a first-order phase transition at $33 \mathrm{GPa}$, on which a volume decrease of about 3\% was found. Rietveld refinements of the XRD data demonstrated the atomic displacements precursory to the phase transition, allowing us to discuss the mechanism of the phase transition. In the Raman experimental data, anomalies were observed in the spectral feature and the guest vibration around 17 GPa. By combining the Raman results with the XRD ones, the vibrational frequency of the guest Ba was investigated as a function of the host cage size. As a result, a linear relation between guest vibrational frequency and the guest-host distance was identified. (c) 2010 American Institute of Physics. [doi:10.1063/1.3276153]
\end{abstract}

\section{INTRODUCTION}

Group IV elements are known to form clathrate structures which consist of nanoscaled polyhedrons encapsulating guest atoms (M) such as $\mathrm{Na}$ and $\mathrm{Ba}^{1,2}$ Among the several types of clathrate structures, the type I clathrates formulated with $\mathrm{M}_{8} \mathrm{IV}_{46}$ are most intensively studied. The group IV atoms can be partially replaced with III or V elements so as to compensate the excess or missing of charges of the guest atoms, resulting in ternary clathrates such as $\mathrm{Ba}_{8} \mathrm{Ga}_{16} \mathrm{Ge}_{30}$. The ternary clathrates $\mathrm{A}_{8} \mathrm{Ga}_{16} \mathrm{Ge}_{30}(\mathrm{~A}=\mathrm{Sr}, \mathrm{Ba}, \mathrm{Eu})$ have been considerably studied ${ }^{3-13}$ as potential candidates adopted to phonon-glass and electron-crystal concept, which is of crucial importance for practical application to thermoelectric devices. Although the low electronic mobility of $\mathrm{Eu}_{8} \mathrm{Ga}_{16} \mathrm{Ge}_{30}$, and the crystal-like thermal conductivity of $\mathrm{Ba}_{8} \mathrm{Ga}_{16} \mathrm{Ge}_{30}$ (Ref. 5) have been reported, these clathrates are still of importance as systems providing rattling vibrations of the guest atoms loosely encapsulated in the clathrate cages. Recently, Takasu et al. ${ }^{12,13}$ have reported, by using Raman spectroscopy, anomalous behaviors in the temperature dependence of the guest vibrations for various clathrate compounds, e.g., $\mathrm{Ba}_{8} \mathrm{Ga}_{16} \mathrm{Ge}_{30}$ and $\mathrm{Sr}_{8} \mathrm{Ga}_{16} \mathrm{Ge}_{30}$. According to their work, the sizes of guest atom and the host cage are dominantly important for the rattling nature of the guest vibration. In order to investigate the properties of the rattling vibration, it is straightforward to perform a study in which the cage size is systematically changed by applying pressure. To do that, the structural and vibrational studies should be simultaneously performed under high pressure.

\footnotetext{
a) Author to whom correspondence should be addressed. Electronic mail: kume@gifu-u.ac.jp.
}

The pressurization also throws light on the structural stability of the clathrate, which is improved by the presence of the guest atoms, as known in the case of Si clathrates. The clathrate structure with $s p^{3}$ bonding network is preserved up to very high pressure, e.g., $40 \mathrm{GPa}$ for the Ba doped type-I Si clathrate $\left(\mathrm{Ba}_{8} \mathrm{Si}_{46}\right)$, while the diamond $\mathrm{Si}(\mathrm{d}-\mathrm{Si})$ transforms to the $\beta$-tin structure around $11 \mathrm{GPa}^{14,15}$ Instead of the structural change, the doped Si clathrates undergo an isostructural phase transition, ${ }^{16-25}$ which has been first observed in the case of $\mathrm{Ba}_{8} \mathrm{Si}_{46}{ }^{16,21,22}$ At $\sim 15 \mathrm{GPa}, \mathrm{Ba}_{8} \mathrm{Si}_{46}$ shows a reversible phase transition on which the unit cell discontinuously decreases in its volume by $5 \%$ (volume collapse) without changes of the crystal structure. It is established that this type of phase transition is commonly observed for the doped type-I $\mathrm{Si}$ clathrates $\left(\mathrm{Ba}_{8} \mathrm{Si}_{46}, \mathrm{~K}_{8} \mathrm{Si}_{46}, \mathrm{I}_{8} \mathrm{Si}_{44} \mathrm{I}_{2}\right.$, $\left.\mathrm{Ba}_{8} \mathrm{Ag}_{6} \mathrm{Si}_{40}, \mathrm{Rb}_{6.15} \mathrm{Si}_{46}\right) .{ }^{16,21,22,25}$ On the other hand, there is no volume-collapse transition in the case of a Ge clathrate $\mathrm{Ba}_{8} \mathrm{Ge}_{43} \square_{3}$ in which there are vacancies on the 6c Wyckoff site in the $P m \overline{3} n$ space group. ${ }^{26}$ According to a theoretical study on $\mathrm{Ba}_{8} \mathrm{Si}_{46}$ and the hypothetical $\mathrm{Ba}_{8} \mathrm{Si}_{43} \square_{3},{ }^{27}$ the $6 \mathrm{c}$ site plays an important role in the volume-collapse transition. In order to verify experimentally the role of the $6 \mathrm{c}$ site, we have to make experiments using $\mathrm{Ba}_{8} \mathrm{Ge}_{46}$ clathrate, which cannot be synthesized at present. Recently, for $\mathrm{I}_{8} \mathrm{Sb}_{8} \mathrm{Ge}_{38}$ clathrate without vacancy, the volume-collapse transition was reported, ${ }^{28}$ implying that the existence of vacancy suppresses the volume-collapse transition.

In this work, for the $\mathrm{Ge}$ based ternary clathrate $\mathrm{Ba}_{8} \mathrm{Ga}_{16} \mathrm{Ge}_{30}$ without vacancy, we performed a combined study of high-pressure Raman and x-ray diffraction (XRD) experiments. We report here a distinct first-order phase transition at $33 \mathrm{GPa}$ at which the cell volume discontinuously decreases by $3 \%$. This phase transition was understood as the 
(a) XRD

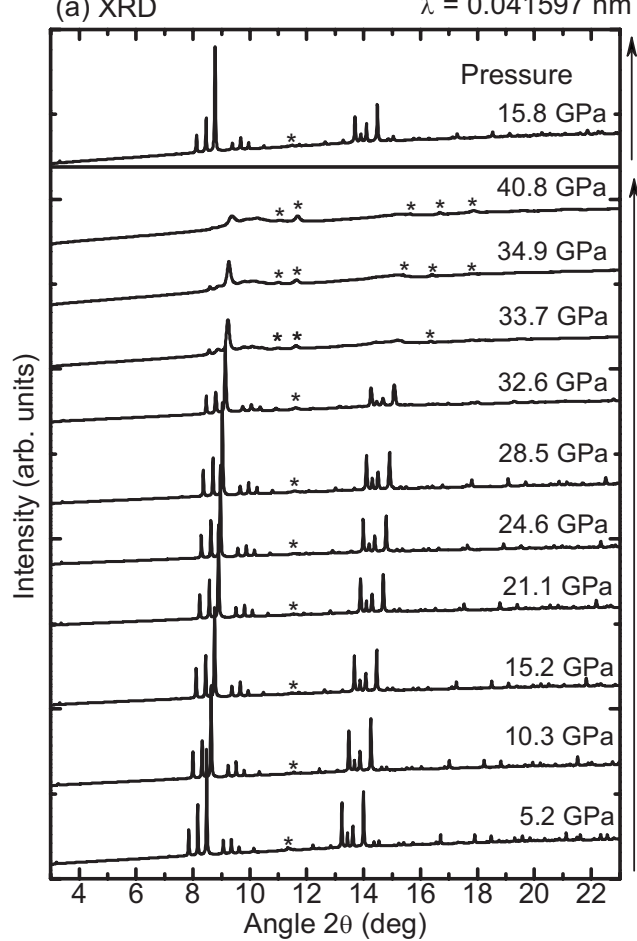

(b) XRD

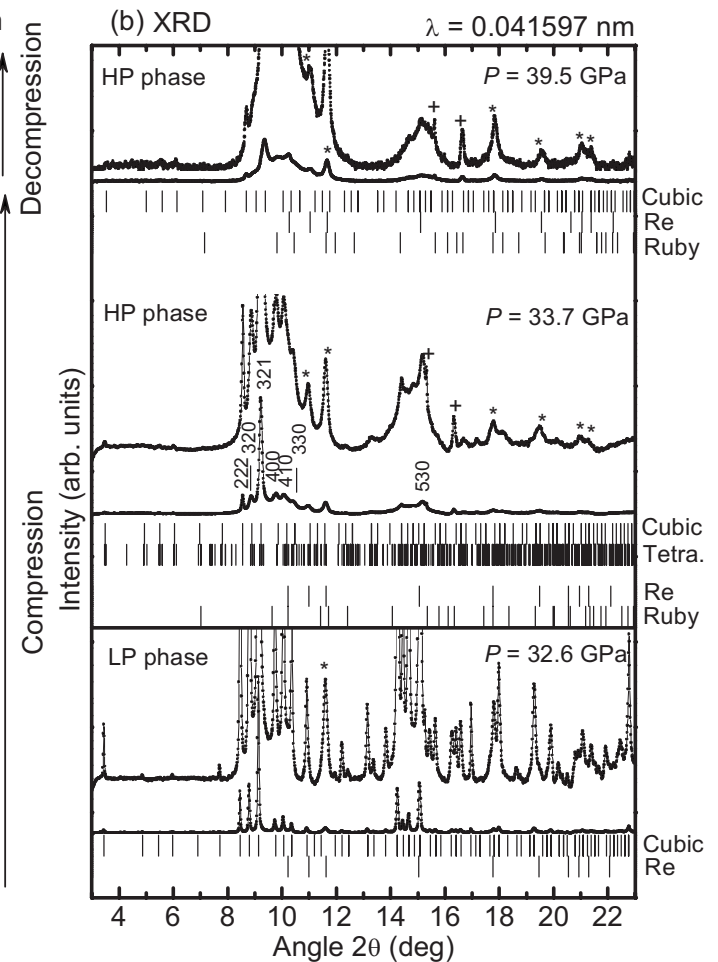

FIG. 1. Synchrotron powder XRD patterns of $\mathrm{Ba}_{8} \mathrm{Ga}_{16} \mathrm{Ge}_{30}$ in the $2 \theta$ range from $3^{\circ}$ to $23^{\circ}$. The pressure medium was helium. In (a), the lower and upper panels correspond to the compression and decompression processes, respectively. The peaks denoted with * are from the Re gasket and ruby. In (b), three diffraction data picked up for discussion of the crystal symmetry of HP phase are presented. The calculated position under simple-cubic and simple tetragonal lattices are indicated with thick marks. The peaks denoted with ${ }^{*}$ and + are, respectively, from the Re gasket and ruby $\left(\alpha-\mathrm{Al}_{2} \mathrm{O}_{3}\right)$, of which the calculated positions (Refs. 31-33) are denoted with thick marks.

similar type of the phase transition observed for $\mathrm{Ba}_{8} \mathrm{Si}_{46} \cdot{ }^{16,21,22}$ In the pressure region lower than $33 \mathrm{GPa}$, the XRD results indicated no sign of phase transition, but the Raman results showed anomalies in the spectral feature and the guest vibrations around $17 \mathrm{GPa}$. The anomalies were discussed through the comparison with the previous results for $\mathrm{Ba}_{8} \mathrm{Ge}_{43} \square_{3}$ or $\mathrm{Ba}_{8} \mathrm{Si}_{46}$. Using the structural data, the guest $\mathrm{Ba}$ vibrational frequency was investigated as a function of the cage size. As a result, a linear relation was found between the frequency and the guest-host distance.

\section{EXPERIMENTAL}

$\mathrm{Ba}_{8} \mathrm{Ga}_{16} \mathrm{Ge}_{30}$ samples were prepared by Ar arc melting methods. After arc melting, specimens were annealed at $790{ }^{\circ} \mathrm{C}$ in vacuum for $12 \mathrm{~h}$ followed either by oil quenching or by furnace cooling. Details for the sample preparation were described elsewhere. ${ }^{11}$ The prepared specimen showed $n$-type conduction. ${ }^{11}$ The high-pressure experiments were carried out by using a diamond anvil cell (DAC) with a metal gasket. The hole made by drilling the metal gasket, serving as the sample chamber, was set to about $100 \mu \mathrm{m}$ in diameter and $50 \mu \mathrm{m}$ in thickness. For Raman measurements, a singlephase sample of $40 \mu \mathrm{m}$ in size was placed into the chamber of the DAC with a ruby chip for pressure measurements. As the pressure-transmitting medium, we used the dense argon that is free from Raman signals. Raman measurements have been performed with an apparatus (JASCO NR1800) which has been improved through detecting the low-frequency Raman signals for various clathrate compounds. ${ }^{18-20,26,29}$ The
$532 \mathrm{~nm}$ line of a solid laser (Coherent Verdi2W) with its intensities of less than $10 \mathrm{~mW}$ was used for the excitation. The spectra were measured with a triple polychromator and a charge-coupled device detector. The resolution of the Raman spectra was about $1 \mathrm{~cm}^{-1}$. To make the powder XRD measurements, the sample of $\mathrm{Ba}_{8} \mathrm{Ga}_{16} \mathrm{Ge}_{30}$ was ground to fine powder, and the powder and a ruby chip were put into the sample chamber of DAC which was prepared in the same manner as that for Raman measurements. The pressure medium was dense argon or helium. Synchrotron powder XRD measurements were carried out with an imaging plate detector installed at the BL10XU beam line of the SPring-8. The camera distance was about $45 \mathrm{~cm}$, and the wavelength of the incident $\mathrm{x}$-ray was $0.041597 \mathrm{~nm}$. The exposure time for obtaining the diffraction pattern was typically $3 \mathrm{~min}$. Structure refinements were carried out using a Rietveld refinement program RIETAN-2000 by Izumi and Ikeda. ${ }^{30}$

\section{RESULTS AND DISCUSSION}

\section{A. Phase transformation at $33 \mathrm{GPa}$ and its mechanism}

Figures 1(a) and 1(b) show the powder XRD patterns obtained for high pressures up to $41 \mathrm{GPa}$. These results were obtained for the He pressure medium. Although the measurements have been carried out using $\mathrm{Ar}$ and $\mathrm{He}$ as pressure media in DAC, there was no significant difference in the experimental results. As seen in Fig. 1(a), an abrupt change of the XRD pattern occurred at $33 \mathrm{GPa}$, at which all the diffraction peaks became broad and weak. In Fig. 1(b) we 


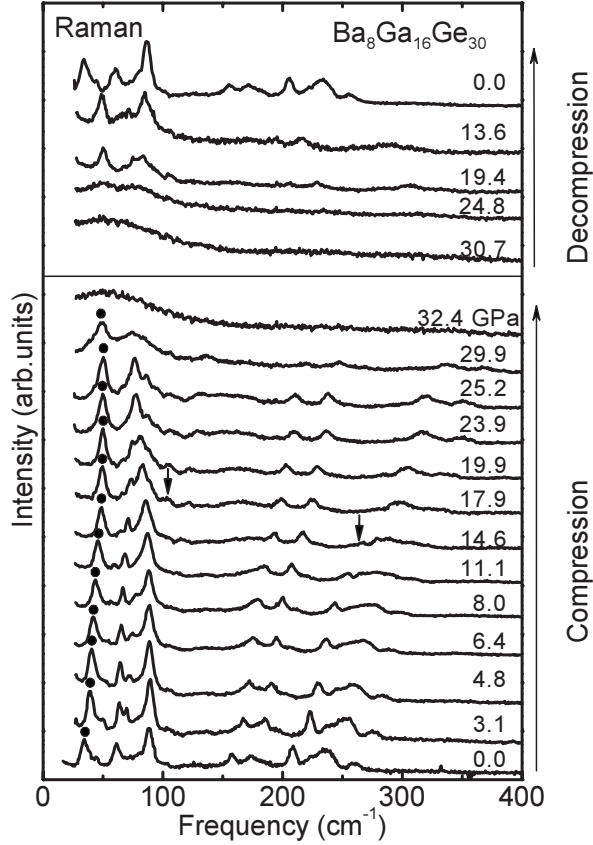

FIG. 2. Raman spectra of $\mathrm{Ba}_{8} \mathrm{Ga}_{16} \mathrm{Ge}_{30}$ obtained for various pressures up to $33 \mathrm{GPa}$ on compression and decompression processes. The perfect reversibility is found on the spectral change. Solid circles correspond to the guest $\mathrm{Ba}$ vibration in the large cage. For arrows denoting the spectra, see text. The pressure medium was argon.

present the XRD patterns for $P=32.6,33.7$, and $39.5 \mathrm{GPa}$, which were picked up for discussion on the phase transition. Above $33 \mathrm{GPa}$, broad bands corresponding to halo patterns appeared around $10^{\circ}$ and $15^{\circ}$. The appearance of halo patterns and the weakening of the diffraction peaks suggest the amorphization. Figure 2 indicates the pressure dependence of Raman spectra which include the peaks from the guest $\mathrm{Ba}$ vibration in the large cage ${ }^{13}$ (solid circles) and the host $\mathrm{Ge} / \mathrm{Ga}$ vibrations in higher frequencies. In the Raman results, all the peaks disappeared and a broad band only was observed above $32 \mathrm{GPa}$. The halo patterns in the XRD (Fig. 1) and the disappearance of the Raman peaks (Fig. 2) suggest again the occurrence of amorphization. However, the amorphization was immediately ruled out because the XRD pattern and Raman peaks were reversibly changed when pressure was released, as shown in the upper panels of Fig. 1(a) and Fig. 2. In general, the amorphization for the covalent materials is irreversible at the room temperature. Actually, the irreversible amorphizations have been observed for other Ge clathrates. ${ }^{26,29}$ As seen in Fig. 2, the pressure giving the Raman-spectral change on decompression $(\sim 20 \mathrm{GPa})$ was much lower than that on compression ( $32 \mathrm{GPa}$ ). This hysteresis demonstrates that the phase transition is of the first order. Therefore, we can conclude that $\mathrm{Ba}_{8} \mathrm{Ga}_{16} \mathrm{Ge}_{30}$ undergoes a reversible first-order phase transformation at $33 \mathrm{GPa}$.

We now discuss the crystal symmetry of the highpressure (HP) phase. As seen in Fig. 1, several XRD peaks were still distinctly observed at the pressures just above 33 $\mathrm{GPa}$, but the number of the observable peaks decreased with increasing pressure. At $39.5 \mathrm{GPa}$, there was no distinct peak in the higher angle region of $2 \theta \geq 11^{\circ}$. Several distinct peaks were from Re gasket ${ }^{31,32}$ or ruby $\left(\alpha-\mathrm{Al}_{2} \mathrm{O}_{3}\right)$ (Ref. 33) which became more remarkable because of low signal intensities of the sample. In order to discuss the crystal symmetry, the observed peaks were indexed with the simple-cubic lattice $(P m \overline{3} n)$ the same as that of the ambient condition. In the low pressure phase at $32.6 \mathrm{GPa}$, all the peaks from the sample were well explained with the cubic symmetry, leading to the lattice constant of $a=0.9775 \mathrm{~nm}$ and the unit-cell volume of $V=0.9342 \mathrm{~nm}^{3}$. On the other hand, in the case of $33.7 \mathrm{GPa}$, almost all the peaks were fitted well with the cubic symmetry, but slight deviations were found between the observed and calculated peak positions of, e.g., the (400) and (410) reflections. The deviations suggest the crystal symmetry different from the cubic. Since the (222) peak did not become broadening and/or splitting on the phase transition in contrast to the other peaks, the tetragonal or orthorhombic distortion that does not split the $(x x x)$ peaks should be considered if the crystal distortion takes place. When we analyzed the data with the tetragonal structure so as to adjust the (222) and (400) peaks, we obtained $a=0.976 \mathrm{~nm}, c=0.945 \mathrm{~nm}$, and $V=0.900 \mathrm{~nm}^{3}$. On the other hand, the assumption of the cubic lattice has deduced $a=0.967 \mathrm{~nm}$ and $V=0.904 \mathrm{~nm}^{3}$. In any cases, the unit-cell volume was found to decrease by $3 \%$ on the phase transition. Although it is difficult to determine here the crystal symmetry because there were only a few observed diffraction peaks, we can identify the phase transition as the volume-collapse phase transition. Since the assumption of the cubic seems not to cause severe disagreements between the calculated and experimental positions of the peaks, apart from the 400 and 410 reflections, for which the XRD peaks from the $\mathrm{Re}^{31,32}$ and ruby $\left(\alpha-\mathrm{Al}_{2} \mathrm{O}_{3}\right)$ (Ref. 33) are probably superimposed, the unit-cell volumes of HP phase were tentatively deduced here under the assumption of the cubic structure.

The change of the XRD pattern on the phase transition allowed us to discuss the mechanism of the volume-collapse phase transition. As shown in Fig. 1(b), at $33 \mathrm{GPa}$, some XRD peaks selectively decreased in intensity, e.g., the 530 reflection peak. The 530 reflection peak corresponds to a $d$ value of which the triple is the same as the distance between the pentagonal planes opposite to each other in the dodecahedral small cage. ${ }^{34}$ Therefore, the disappearance of this peak implies random deformation and/or rotation of the small cages. On the other hand, the 321 peak is distinctly observed still in the HP phase. Taking account of that the 321 reflection dominantly comes from the ordering of $\mathrm{Ba}$ atoms, we see that the long-range order of $\mathrm{Ba}$ atom is still maintained. Therefore, it is considered that the HP phase is formed by the ordered $\mathrm{Ba}$ atoms surrounded with highly distorted $\mathrm{Ge} / \mathrm{Ga}$ host cages. In addition, the reversible behavior of the phase transition allows us to be aware that the clathrate-network bondings are not destroyed on this phase transition. The highly distorted cage can break the $k \sim 0$ selection rule on the Raman scattering, leading to the amorphouslike Raman band in the HP phase (Fig. 2). In the present data of $\mathrm{Ba}_{8} \mathrm{Ga}_{16} \mathrm{Ge}_{30}$, we did not confirm the irreversible amorphization. If the amorphization appears, it will take place, at least, above $40 \mathrm{GPa}$, which is higher than 30-40 GPa in the case of $\mathrm{Ba}_{8} \mathrm{Ge}_{43} \square_{3}$ (Ref. 26) and comparable with the case of $\mathrm{Ba}_{8} \mathrm{Si}_{46} \cdot{ }^{21}$ 


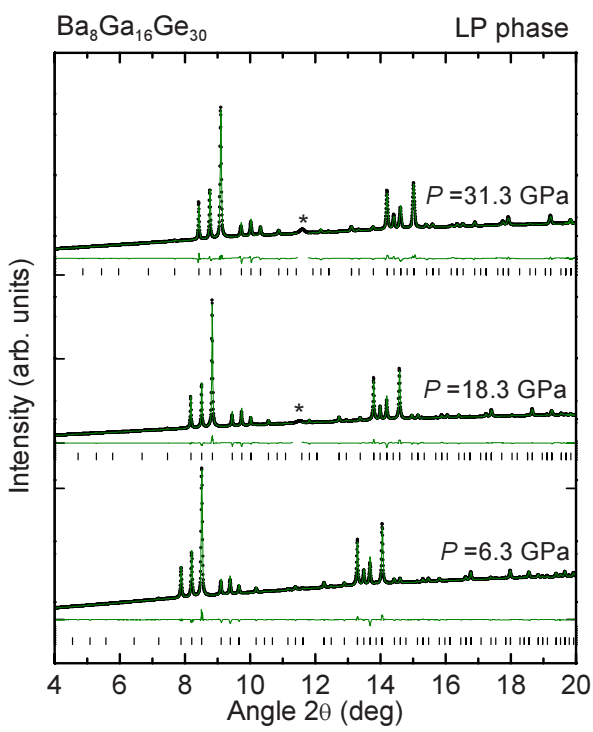

FIG. 3. (Color online) Typical results of Rietveld analyses performed up to $33 \mathrm{GPa}$. Solid circles denote the experimental data; solid lines, the calculated patterns; the thick marks, the calculated peak position; the bottom curves, the differences between the observed and calculated pattern. The angle regions in which the strong gasket peak appears were omitted from the fitting range.

For understanding the volume-collapse transition at 33 $\mathrm{GPa}$, it is straightforward to determine the atomic position before and after the phase transition. Since, however, the atomic positions were not available in the distorted HP phase, we present here the pressure induced atomic displacements precursory to the phase transition for discussing the mechanism of the phase transition. The pressure evolution of the atomic position was obtained from the Rietveld analyses which ware performed for all the pressures studied here, using the split pseudo-Voigt function of Toraya as the profile function. As seen in Fig. 3, the Rietveld refinements were successfully done up to $33 \mathrm{GPa}$. The fitting parameters related with the crystal structure were the lattice constant, the inner atomic coordinates of $\mathrm{Ge} / \mathrm{Ga}$ at $16 \mathrm{i}$ and $24 \mathrm{k}$ sites, and Debye-Waller factors [isotropic atomic displacement parameters (ADPs)] of Ba at 2a and 6d sites. All the site occupations were fixed as 0.979 for $\mathrm{Ba}(2 \mathrm{a}), 0.971$ for $\mathrm{Ba}(6 \mathrm{~d}), 0.955$ for $\mathrm{Ge} / \mathrm{Ga}(6 \mathrm{c}), 1.000$ for $\mathrm{Ge} / \mathrm{Ga}(16 \mathrm{i}, 24 \mathrm{k})$ and the ADPs of $\mathrm{Ge} / \mathrm{Ga}(6 \mathrm{c}, 16 \mathrm{i}$, and $24 \mathrm{k})$ were also fixed to $107 \mathrm{pm}^{2}$, according to the data obtained at the ambient condition in the pre-

TABLE I. Typical results of Rietveld refinements for $\mathrm{Ba}_{8} \mathrm{Ga}_{16} \mathrm{Ge}_{30}$ under high pressures. The space group is $P m \overline{3} n$ and atom positions are $\mathrm{Ba}(2 \mathrm{a})$ $0,0,0 ; \mathrm{Ba}(6 \mathrm{~d}) 1 / 4,1 / 2,0 ; \mathrm{Ge} / \mathrm{Ga}(6 \mathrm{c}) 1 / 4,0,1 / 2 ; \mathrm{Ge} / \mathrm{Ga}(16 \mathrm{i})(x, x, x)$; and $\mathrm{Ge} /$ $\mathrm{Ga}(24 \mathrm{k})(0, y, z)$.

\begin{tabular}{lccc}
\hline \hline Pressure $(\mathrm{GPa})$ & 6.3 & 18.3 & 31.3 \\
$R_{\mathrm{wp}}(\%)$ & 1.41 & 1.78 & 2.04 \\
$R_{\mathrm{p}}(\%)$ & 0.67 & 0.94 & 1.12 \\
Lattice parameter $(\mathrm{nm})$ & $10.4781(3)$ & $10.1010(2)$ & $9.8093(3)$ \\
$\mathrm{ADP}[\mathrm{Ba}(2 \mathrm{a})]\left(\mathrm{pm}^{2}\right)$ & $54(9)$ & $93(9)$ & $60(11)$ \\
$\mathrm{ADP}[\mathrm{Ba}(6 \mathrm{~d})]\left(\mathrm{pm}^{2}\right)$ & $394(12)$ & $335(12)$ & $93(12)$ \\
$x[\mathrm{Ge} / \mathrm{Ga}(16 \mathrm{i})]$ & $0.1841(1)$ & $0.1837(1)$ & $0.1824(2)$ \\
$y[\mathrm{Ge} / \mathrm{Ga}(24 \mathrm{k})]$ & $0.3106(2)$ & $0.3119(2)$ & $0.3140(3)$ \\
$z[\mathrm{Ge} / \mathrm{Ga}(24 \mathrm{k})]$ & $0.1205(2)$ & $0.1194(2)$ & $0.1197(2)$ \\
\hline \hline
\end{tabular}

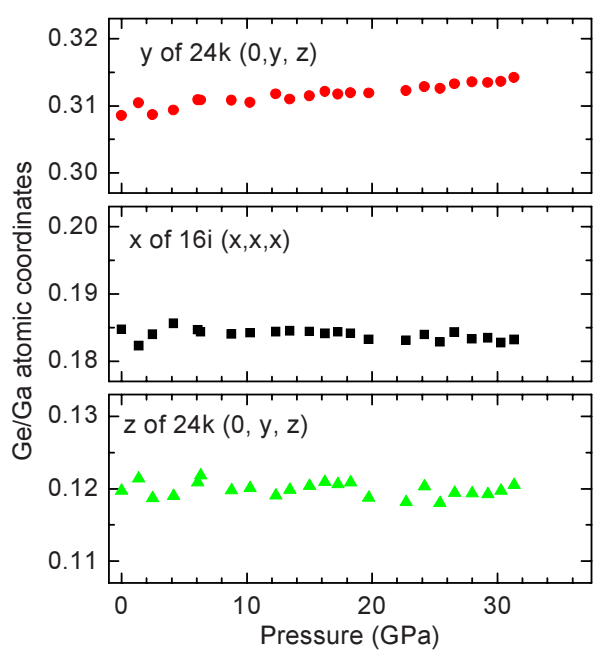

FIG. 4. (Color online) Pressure dependence of internal atomic coordinate parameters obtained by Rietveld refinements.

vious work. ${ }^{11}$ Furthermore, it was assumed that $\mathrm{Ge}$ and $\mathrm{Ga}$ randomly distribute on each sites (6c, 16i, and 24k) with the stoichiometric ratio of 30:16. Typical results of the analyses are summarized in Table I. Figure 4 shows the pressure dependence of the internal coordinates of the host atoms obtained from the Rietveld refinement of XRD data. Since the internal atomic coordinates for the type I clathrate are $(0,0,0)$ for $\mathrm{Ba}(2 \mathrm{a}),(0.25,0.5,0)$ for $\mathrm{Ba}(6 \mathrm{~d}),(0.25,0,0.5)$ for $\mathrm{Ge} /$ $\mathrm{Ga}(6 \mathrm{c}),(x, x, x)$ for $\mathrm{Ge} / \mathrm{Ga}(16 \mathrm{i})$, and $(0, y, z)$ for $\mathrm{Ge} / \mathrm{Ga}(24 \mathrm{k})$, there are three parameters for the atomic coordinates ( $y$ and $z$ of $24 \mathrm{k}$ and $x$ of $16 \mathrm{i}$ site). As seen in this figure, only the $y$ coordinate of $24 \mathrm{k}$ site slightly increased with increasing pressure, while the others almost kept constant. This implies that there are some parts favorably shrinking when the crystal is compressed. In Fig. 5, we depicted the displacements of the $24 \mathrm{k}$ atoms using the arrows, according to the results shown in Fig. 5. These displacements give rise to the deformation of the hexagonal ring in the large cage.

The deformation of the hexagonal ring reminds us about Sn clathrates with vacancies on the $6 \mathrm{c}$ site, which is partially occupied by $\mathrm{Sn}$ atom. According to a recent work on

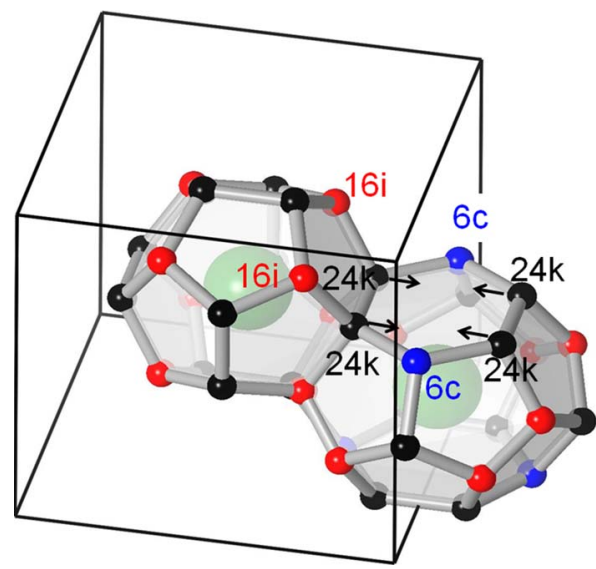

FIG. 5. (Color online) Cage structure of $\mathrm{Ba}_{8} \mathrm{Ga}_{16} \mathrm{Ge}_{30}$. The unit cell is represented by a cube drawn by solid lines. The Wyckoff sites (6c, 16i, and $24 \mathrm{k}$ ) of the host atoms are indicated. Arrows shows the displacements for the $24 \mathrm{k}$ site induced by pressurization. 


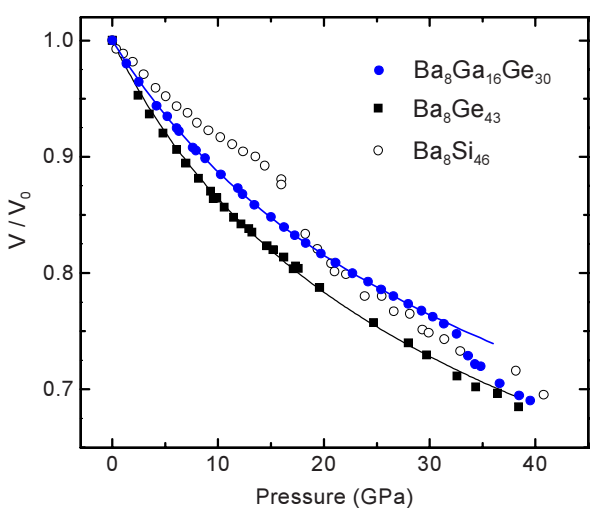

FIG. 6. (Color online) Pressure dependence of unit-cell volumes of $\mathrm{Ba}_{8} \mathrm{Ga}_{16} \mathrm{Ge}_{30}, \mathrm{Ba}_{8} \mathrm{Ge}_{43} \square_{3}$ (Ref. 26), and $\mathrm{Ba}_{8} \mathrm{Si}_{46}$ (Ref. 21) normalized with those under the ambient condition. Symbols correspond to the experimental data, and lines the result of theoretical fitting with Murnaghan's EOS.

$\mathrm{Rb}_{8} \mathrm{Sn}_{44} \square_{2}$ (Ref. 35), the $\mathrm{Sn}$ atoms surrounding the vacancy (6c site) are slightly displaced toward the vacancy, resulting in the deformation of the hexagonal ring. Conversely, the deformation of the hexagonal ring tends to make the vacancy on the $6 \mathrm{c}$ site. The vacancy generation is able to reduce the cell volume. In fact, in spite of the almost the same atomic radii for $\mathrm{Ge}$ and $\mathrm{Ga}, \mathrm{Ba}_{8} \mathrm{Ge}_{43} \square_{3}$ takes a smaller volume than $\mathrm{Ba}_{8} \mathrm{Ga}_{16} \mathrm{Ge}_{30}$. As has been suggested theoretically, ${ }^{27}$ the vacancy generation on the $6 \mathrm{c}$ site is a candidate for the mechanism of the volume-collapse phase transition. However, the escape of the $6 \mathrm{c}$ atoms from the crystal should be ruled out because of the reversibility of the phase transition observed in the present results (Figs. 1 and 2). Consequently, the following scenario can be considered for the volume-collapse phase transition. At first, the hexagonal ring is deformed under the high pressure. The $6 \mathrm{c}$ atoms are largely displaced from the initial position. This leads to a deformation of the large cage. Simultaneously, the rotation and/or deformation of the small cages takes place because the $6 \mathrm{c}$ atom bridges the small cages. As a result, a highly distorted cage structure is realized.

\section{B. Volume change associated with the phase transition and equation of state (EOS)}

In Fig. 6, we plotted the pressure dependence of the unit-cell volume deduced from the XRD results under the $P m \overline{3} n$ structure. For HP phase above $33 \mathrm{GPa}$, the volume was obtained by using (222) and (321) reflections (see Fig. 1) under the assumption of the cubic structure. In this figure, the volume relative to that obtained at the ambient condition $\left(V / V_{0}\right)$ was plotted as a function of pressure. For comparison, the previous data obtained for $\mathrm{Ba}_{8} \mathrm{Si}_{46}$ (Ref. 21) and $\mathrm{Ba}_{8} \mathrm{Ge}_{43} \square_{3}$ (Ref. 26) were also presented. For $\mathrm{Ba}_{8} \mathrm{Ga}_{16} \mathrm{Ge}_{30}$, the $3 \%$ volume decrease was found on the phase transition, similarly to the case of $\mathrm{Ba}_{8} \mathrm{Si}_{46}$. In contrast, for $\mathrm{Ba}_{8} \mathrm{Ge}_{43} \square_{3}$ there is no volume-collapse transition. According to the recent paper on $\mathrm{I}_{8} \mathrm{Sb}_{8} \mathrm{Ge}_{38},{ }^{28}$ the volume-collapse transition was observed as a kink in the pressure dependence of the volume at $42 \mathrm{GPa}$. The findings of the volume-collapse phase transitions of $\mathrm{Ba}_{8} \mathrm{Ga}_{16} \mathrm{Ge}_{30}$ and $\mathrm{I}_{8} \mathrm{Sb}_{8} \mathrm{Ge}_{38}$ suggest that the occurrence of this phase transition is independent of
TABLE II. Isothermal bulk moduli $B_{0}$ and its pressure derivative $B^{\prime}$ obtained by Murnaghan's EOS.

\begin{tabular}{|c|c|c|c|}
\hline Phase & $B_{0}(\mathrm{GPa})$ & $B^{\prime}$ & \\
\hline $\mathrm{Ba}_{8} \mathrm{Ga}_{16} \mathrm{Ge}_{30}{ }^{\mathrm{a}}$ & 67.2 & 3.5 & Expt. \\
\hline $\mathrm{Ba}_{8} \mathrm{Ge}_{43} \square_{3}^{\mathrm{b}}$ & 52.8 & 3.4 & Expt. \\
\hline $\mathrm{I}_{8} \mathrm{Sb}_{8} \mathrm{Ge}_{38} \mathrm{c}$ & 64.7 & 4.5 & Expt. \\
\hline $\mathrm{Ge}_{46}{ }^{\mathrm{d}}$ & 61.3 & 4.8 & Calc. \\
\hline $\mathrm{d}-\mathrm{Ge}^{\mathrm{e}}$ & 74.9 & 3.0 & Expt. \\
\hline $\mathrm{Ba}_{8} \mathrm{Si}_{46}{ }^{\mathrm{f}}$ & 93 & & Expt. \\
\hline $\mathrm{Si}_{46}{ }^{\mathrm{f}}$ & 87 & & Calc. \\
\hline $\mathrm{d}-\mathrm{Si}^{\mathrm{g}}$ & 99.9 & 3.8 & Expt. \\
\hline
\end{tabular}

${ }^{\mathrm{a}}$ Present work.

${ }^{\mathrm{b}}$ Reference 26 .

${ }^{\mathrm{c}}$ Reference 28 .

${ }^{\mathrm{d}}$ Reference 37.

the guest atoms and the substitution of the host atoms. Taking into account the fact that $\mathrm{Si}$ calthrates $\left(\mathrm{Ba}_{8} \mathrm{Si}_{46}, \mathrm{~K}_{8} \mathrm{Si}_{46}, \mathrm{I}_{8} \mathrm{Si}_{44} \mathrm{I}_{2}, \mathrm{Ba}_{8} \mathrm{Ag}_{6} \mathrm{Si}_{40}, \mathrm{Rb}_{6.15} \mathrm{Si}_{46}\right)$ have indicated the volume-collapse phase transitions independently of the guest and the substitution of the host, the isostructural volume-collapse phase transition is observed as a common properties through the $\mathrm{Si}$ and Ge clathrates. The only exception has been observed for $\mathrm{Ba}_{8} \mathrm{Ge}_{43} \square_{3}$. This strongly suggests that the existence of vacancies suppresses the volume-collapse phase transition.

Solid curves in Fig. 6 represent EOS fitted to the experimental data using the Murnaghan's equation ${ }^{36}$

$$
\frac{V}{V_{0}}=\left[\frac{B_{0}+B^{\prime} p}{B_{0}+B^{\prime} p_{0}}\right]^{-1 / B^{\prime}},
$$

where $V_{0}$ is the volume at pressure $p_{0}$, and $B_{0}$ and $B^{\prime}$ are the bulk modulus and its pressure derivative, respectively. Here, $V_{0}$ and $p_{0}$ in the case of $\mathrm{Ba}_{8} \mathrm{Ga}_{16} \mathrm{Ge}_{30}$ are $1.24789 \mathrm{~nm}^{3}$ and 0 $\mathrm{GPa}$, respectively. The pressure range used for fitting was 0-30 GPa for the present $\mathrm{Ba}_{8} \mathrm{Ga}_{16} \mathrm{Ge}_{30}$. As seen in Fig. 6, the fitted curve reproduced well the experimental one, indicating no sign of phase transition up to $30 \mathrm{GPa}$. The fitting parameters $\left(B_{0}\right.$ and $\left.B^{\prime}\right)$ are summarized in Table II, together with the previous data reported for other clathrates and the d-Si and diamond germanium (d-Ge). From the comparison of the bulk moduli $B_{0}$ between $\mathrm{Ba}_{8} \mathrm{Ge}_{43}$ and the other $\mathrm{Ge}$ based clathrates $\left(\mathrm{Ba}_{8} \mathrm{Ga}_{16} \mathrm{Ge}_{30}, \mathrm{I}_{8} \mathrm{Sb}_{8} \mathrm{Ge}_{38}\right)$, it can be seen that the absence of the host atoms of $6 \mathrm{c}$ site makes the structure considerably compressible, which is compatible with the calculated EOSs of $\mathrm{Ba}_{8} \mathrm{Si}_{46}$ and hypothetic $\mathrm{Ba}_{8} \mathrm{Si}_{43} \square_{3}$ with vacancies in the $6 \mathrm{c}$ site. ${ }^{27}$

\section{Raman spectral change and guest vibration in the lower pressure region}

In Fig. 7, Raman peak frequencies are plotted as a function of pressure. For the type I clathrate without any defects and disorders, the first-order Raman-active modes are predicted by the group theory to have $3 A_{1 g}+8 E_{g}+9 T_{2 g}$ symmetries (20 peaks, 46 modes). From Fig. 7, we can identify 14 vibrational modes. Among them, the two modes in the lowest-frequency region correspond to the guest modes in the large cage, and can be assigned to $E_{g}$ and $T_{2 g}$ modes. The 


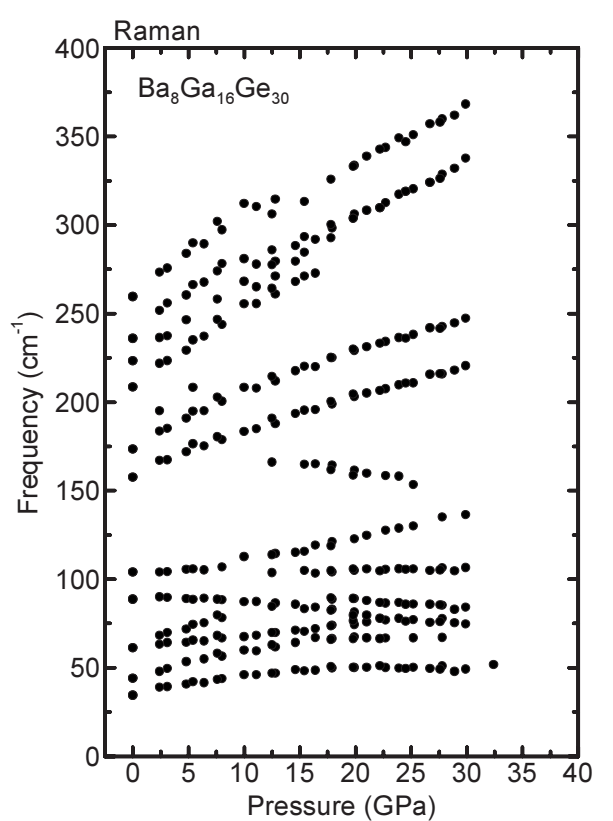

FIG. 7. Raman frequencies of $\mathrm{Ba}_{8} \mathrm{Ga}_{16} \mathrm{Ge}_{30}$ as a function of pressure.

$T_{2 g}$ mode, of which the vibrational direction is perpendicular to the $4_{2}$ axis of the large cage, is known to be located at the lowest frequency. ${ }^{13}$ In the present results obtained at $1 \mathrm{~atm}$, $T_{2 g}$ and $E_{g}$ modes were observed at 35 and $44 \mathrm{~cm}^{-1}$, respectively. The other modes located at higher frequencies correspond to the host cage vibrations. It should be noted here that there are softening modes in the host vibrations; the vibrations of 89 and $104 \mathrm{~cm}^{-1}$ at 1 atm shifted to lower frequencies with increasing pressure. These softening behaviors have been commonly observed for the other clathrates; vibrational modes at $80 \mathrm{~cm}^{-1}$ of $\mathrm{Ba}_{8} \mathrm{Ge}_{43}{ }^{26}{ }^{26}$ at $153 \mathrm{~cm}^{-1}$ of $\mathrm{Ba}_{8} \mathrm{Si}_{46}{ }^{18}$, at $177 \mathrm{~cm}^{-1}$ of $\mathrm{K}_{8} \mathrm{Si}_{46}{ }^{20}{ }^{20}$ at $133 \mathrm{~cm}^{-1}$ of $\mathrm{I}_{8} \mathrm{Si}_{44} \mathrm{I}_{2},{ }^{19}$ at $186 \mathrm{~cm}^{-1}$ of $\mathrm{Rb}_{6.15} \mathrm{Si}_{46},{ }^{25}$ and at $134 \mathrm{~cm}^{-1}$ of $\mathrm{Si}_{136}$ clathrate ${ }^{40}$ measured by Raman experiments. According to the previous discussion, ${ }^{20,25,26}$ the softening modes originate from shear modes corresponding to zone-boundary TA phonons in the diamond structure, which are known to show a softening with pressure. ${ }^{41}$ Therefore, the softening modes in the clathrates arise from the $s p^{3}$ tetrahedral bonding nature. In Fig. 7, we also note that the mode of $166 \mathrm{~cm}^{-1}$ at 11 $\mathrm{GPa}$ shows the softening. This mode is interpreted with the overtone mode because the frequencies were almost twice as large as those of the mode of $84 \mathrm{~cm}^{-1}$ at $11 \mathrm{GPa}$ over the range from 11 to $25 \mathrm{GPa}$.

In Fig. 7, we also note that, for the high-frequency modes located around $200-250 \mathrm{~cm}^{-1}$ at $1 \mathrm{~atm}$, the number of the observed peaks decreased at $15 \mathrm{GPa}$. This is not attributed to the change in the crystal symmetry, but to the change of the Raman intensities because there is no structural transformation in this pressure region. Actually, in Fig. 2 , the gradual intensity changes were clearly observed for the peaks denoted with arrows; the $100 \mathrm{~cm}^{-1}\left(250 \mathrm{~cm}^{-1}\right)$ peaks gradually increased (decreased) in intensity around $15 \mathrm{GPa}$. Taking into account the fact that the Raman intensity is sensitive to electron polarizability, a change in the electronic distribution is considered to occur. We also note anomalous behavior on the guest Ba vibration around 17 GPa in Fig. 7.

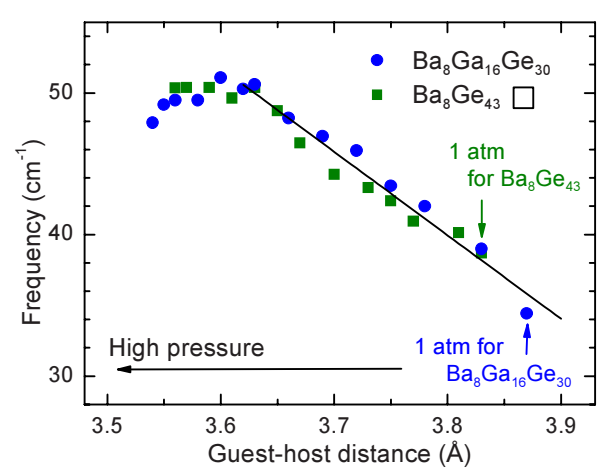

FIG. 8. (Color online) The vibration frequencies of Ba encapsulated in the large cages of the Ge clathrates against the guest-host distance. Solid squares and circles correspond to $\mathrm{Ba}_{8} \mathrm{Ga}_{16} \mathrm{Ge}_{30}$ and $\mathrm{Ba}_{8} \mathrm{Ge}_{43} \square_{3}$, respectively. The straight line is a fitted line under the assumption of a linear function of the distance of the guest-host distance, using both the data in the range higher than $0.362 \mathrm{~nm}$.

The lowest-frequency mode (the mode of $\mathrm{Ba}$ in the large cage) shifted to higher frequencies with increasing pressure up to $\sim 17 \mathrm{GPa}$, and then to lower frequencies in the higher pressure region $>20 \mathrm{GPa}$, in spite of the $\mathrm{Ba}$ atoms more tightly confined in the cages at higher pressures. For understanding these anomalies, there are hints in the previous studies on $\mathrm{Ba}_{8} \mathrm{Si}_{46}$ (Refs. 18 and 24). According to the study by Tse et al. ${ }^{24}$ the electronic topology around the Ba in large cage starts to change around $7 \mathrm{GPa}$ due to the charge transfer. Correspondingly, $\mathrm{Ba}_{8} \mathrm{Si}_{46}$ shows changes in the Raman intensities for the $\mathrm{Ba}$ and $\mathrm{Si}$ vibrations around $7 \mathrm{GPa}{ }^{18}$ Therefore, the charge transfer can change the Raman relative intensity. The frequency softening of $\mathrm{Ba}$ in the present results can be interpreted in terms of the guest-host interaction weakened by the charge transfer. In order to clarify it, it is required to carry out, e g., the measurements of the reflectivity, the electronic conductivity, or maximum entropy method (MEM) analyses leading to the electronic topology.

Recently, Takasu et al. ${ }^{12,13}$ have reported that the sizes of the guest atoms and the space in the host cage are crucially important for the guest vibrational frequency. Thus, it is worthwhile investigating the guest vibration as a function of host cage size. Figure 8 shows the Ba vibrational frequency plotted as a function of the averaged distance between the guest and host atoms. For comparison, the results previously obtained for $\mathrm{Ba}_{8} \mathrm{Ge}_{43} \square_{3}$ are also presented. It should be noted that both of the results on $\mathrm{Ba}_{8} \mathrm{Ga}_{16} \mathrm{Ge}_{30}$ and $\mathrm{Ba}_{8} \mathrm{Ge}_{43} \square_{3}$ trace an identical curve. In the region of relatively large distance (in the lower pressures), the frequency is linearly changed. Therefore, the spring constant (proportional to the square of the frequency) is to be proportional to the square of the guest-host distance. This suggests that the guest atoms in the large cage feel a quartic unharmonic potential, which is well consistent with the previous discussion made for the temperature dependence of $\mathrm{Ba}$ vibration of $\mathrm{Ba}_{8} \mathrm{Ga}_{16} \mathrm{Ge}_{30}$ by Takasu et al. ${ }^{12}$ It should be stressed here that $\mathrm{Ba}$ vibrations in $\mathrm{Ba}_{8} \mathrm{Ge}_{43} \square_{3}$ and $\mathrm{Ba}_{8} \mathrm{Ga}_{16} \mathrm{Ge}_{30}$ indicate the identical behavior. Therefore, the difference of the $\mathrm{Ba}$ frequencies observed at 1 atm in between $\mathrm{Ba}_{8} \mathrm{Ga}_{16} \mathrm{Ge}_{30}\left(34 \mathrm{~cm}^{-1}\right)$ and $\mathrm{Ba}_{8} \mathrm{Ge}_{43} \square_{3}\left(39 \mathrm{~cm}^{-1}\right)$ is explained by the difference of the cage size at $1 \mathrm{~atm}$. We also 
note that, when the guest-host distance decreases to a critical value $\left(0.36 \mathrm{~nm}\right.$ corresponding to $\sim 17 \mathrm{GPa}$ for $\mathrm{Ba}_{8} \mathrm{Ga}_{16} \mathrm{Ge}_{30}$, $\sim 13 \mathrm{GPa}$ for $\mathrm{Ba}_{8} \mathrm{Ge}_{43} \square_{3}$ ), the frequency starts to decrease. It is considered that the guest-host charge transfer mentioned above begins at the critical distance.

\section{CONCLUSION}

In this paper, we presented a combined study of highpressure Raman and XRD experiments performed on $\mathrm{Ba}_{8} \mathrm{Ga}_{16} \mathrm{Ge}_{30}$. We have identified the volume-collapse phase transition at $33 \mathrm{GPa}$, on which the discontinuous volume decrease of $3 \%$ was found. From the reversible behavior with hysteresis, this phase transition was found to be of the first order. The Rietveld analyses strongly suggested that the $6 \mathrm{c}$ site in the clathrate has main role in the volume-collapse transition. In the pressure region lower than $33 \mathrm{GPa}$, the Raman spectra indicated anomalies around $17 \mathrm{GPa}$, in spite of no anomalies in the XRD results, implying a change in the electronic distribution by charge transfer between the guest and host cage. The Ba vibrations were investigated as a function of the host cage size. A linear relation between the $\mathrm{Ba}$ vibration and the guest-host distance holds in $\mathrm{Ba}_{8} \mathrm{Ga}_{16} \mathrm{Ge}_{30}$ and $\mathrm{Ba}_{8} \mathrm{Ge}_{43} \square_{3}$.

\section{ACKNOWLEDGMENTS}

This work was carried out under KAKENHI (Grant-inAid for Scientific Research) on the Priority Areas "New Materials Science Using Regulated Nano Spaces-Strategy in Ubiquitous Elements" from the Ministry of Education, Culture, Sports, Science and Technology of Japan. The synchrotron radiation experiments were performed at the SPring- 8 with the approval of the Japan Synchrotron Radiation Research Institute (JASRI) (Proposal No. 2007B1633). Figure 5 was drawn with the aid of VENUS developed by Dilanian and Izumi.

${ }^{1}$ J. S. Kasper, P. Hagenmuller, M. Pouchard, and C. Cros, Science 150, 1713 (1965).

${ }^{2}$ S. Bobev and S. C. Sevov, J. Solid State Chem. 153, 92 (2000) and references therein.

${ }^{3}$ J. L. Cohn, G. S. Nolas, V. Fessatidis, T. H. Metcalf, and G. A. Slack, Phys. Rev. Lett. 82, 779 (1999).

${ }^{4}$ J. F. Meng, N. V. C. Sheker, J. V. Badding, and G. S. Nolas, J. Appl. Phys. 89, 1730 (2001).

${ }^{5}$ B. C. Sales, B. C. Chakoumakos, R. Jin, J. R. Thompson, and D. Mandrus, Phys. Rev. B 63, 245113 (2001).

${ }^{6}$ S. Paschen, W. Carrillo-Cabrera, A. Bentien, V. H. Tran, M. Baenitz, Yu. Grin, and F. Steglich, Phys. Rev. B 64, 214404 (2001).

${ }^{7}$ C. Gatti, L. Bertini, N. P. Blake, and B. B. Iversen, Chemistry 9, 4556 (2003).

${ }^{8}$ R. P. Hermann, W. Schweika, O. Leupold, R. Ruffer, G. S. Nolas, F. Grandjean, and G. J. Long, Phys. Rev. B 72, 174301 (2005).
${ }^{9}$ R. P. Hermann, V. Keppens, P. Bonville, G. S. Nolas, F. Grandjean, G. J. Long, H. M. Christen, B. C. Chakoumakos, B. C. Sales, and D. Mandrus, Phys. Rev. Lett. 97, 017401 (2006).

${ }^{10}$ M. Christensen, N. Lock, J. Overgaard, and B. B. Iversen, J. Am. Chem. Soc. 128, 15657 (2006).

${ }^{11}$ N. L. Okamoto, K. Kishida, K. Tanaka, and H. Inui, J. Appl. Phys. 100, 073504 (2006).

${ }^{12}$ Y. Takasu, T. Hasegawa, N. Ogita, M. Udagawa, M. A. Avila, K. Suekuni, I. Ishii, T. Suzuki, and T. Takabatake, Phys. Rev. B 74, 174303 (2006).

${ }^{13}$ Y. Takasu, T. Hasegawa, N. Ogita, M. Udagawa, M. A. Avila, K. Suekuni, and T. Takabatake, Phys. Rev. Lett. 100, 165503 (2008).

${ }^{14}$ D. Olego and M. Cardona, Phys. Rev. B 25, 1151 (1982).

${ }^{15}$ Z. Sui, H. H. Burke, and I. P. Herman, Phys. Rev. B 48, 2162 (1993).

${ }^{16}$ A. San-Miguel, P. Melinon, D. Connetable, X. Blase, F. Tournus, E. Reny, S. Yamanaka, and J. P. Itie, Phys. Rev. B 65, 054109 (2002).

${ }^{17}$ J. S. Tse, S. Desgreniers, Z. Q. Li, M. R. Ferguson, and Y. Kawazoe, Phys. Rev. Lett. 89, 195507 (2002).

${ }^{18}$ T. Kume, H. Fukuoka, T. Koda, S. Sasaki, H. Shimizu, and S. Yamanaka, Phys. Rev. Lett. 90, 155503 (2003).

${ }^{19}$ H. Shimizu, T. Kume, T. Kuroda, S. Sasaki, H. Fukuoka, and S. Yamanaka, Phys. Rev. B 68, 212102 (2003).

${ }^{20}$ T. Kume, T. Koda, S. Sasaki, H. Shimizu, and J. S. Tse, Phys. Rev. B 70, 052101 (2004)

${ }^{21}$ A. San Miguel, A. Merlen, P. Toulemonde, T. Kume, S. Le Floch, A. Aouizerat, S. Pascarelli, G. Aquilanti, O. Mathon, T. Le Bihan, J.-P. Itié, and S. Yamanaka, Europhys. Lett. 69, 556 (2005).

${ }^{22}$ A. San Miguel and P. Toulemonde, High Press. Res. 25, 159 (2005).

${ }^{23}$ L. Yang, Y. M. Ma, T. Iitaka, J. S. Tse, K. Stahl, Y. Ohishi, Y. Wang, R. W. Zhang, J. F. Liu, H.-K. Mao, and J. Z. Jiang, Phys. Rev. B 74, 245209 (2006).

${ }^{24}$ J. S. Tse, R. Flacau, S. Desgreniers, T. Iitaka, and J. Z. Jiang, Phys. Rev. B 76, 174109 (2007)

${ }^{25}$ D. Machon, P. Toulemonde, P. F. McMillan, M. Amboage, A. Muñoz, P. Rodríguez-Hernández, and A. San-Miguel, Phys. Rev. B 79, 184101 (2009).

${ }^{26}$ H. Shimizu, T. Iitaka, T. Fukushima, T. Kume, S. Sasaki, N. Sata, Y. Ohishi, H. Fukuoka, and S. Yamanaka, J. Appl. Phys. 101, 063549 (2007).

${ }^{27}$ T. Iitaka, Phys. Rev. B 75, 012106 (2007).

${ }^{28}$ H. Shimizu, R. Oe, S. Ohno, T. Kume, S. Sasaki, K. Kishimoto, T. Koyanagi, and Y. Ohishi, J. Appl. Phys. 105, 043522 (2009).

${ }^{29}$ H. Shimizu, T. Fukushima, T. Kume, S. Sasaki, H. Fukuoka, and S. Yamanaka, J. Appl. Phys. 101, 113531 (2007).

${ }^{30}$ F. Izumi and T. Ikeda, Mater. Sci. Forum 321-324, 198 (2000).

${ }^{31}$ Y. K. Vohra, S. J. Duclos, and A. L. Ruoff, Phys. Rev. B 36, 9790 (1987).

${ }^{32}$ L. G. Liu, T. Takahashi, and W. A. Bassett, J. Phys. Chem. Solids 31, 1345 (1970).

${ }^{33}$ L. S. Dubrovinsky, S. K. Saxena, and P. Lazor, Phys. Chem. Miner. 25, 434 (1998)

${ }^{34}$ When the atomic coordinates of the $24 \mathrm{k}$ and $16 \mathrm{i}$ sites are $(0,0.30,0.12)$ and $(0.1875,0.1875,0.1875)$, respectively, the $\{530\}$ planes have the $d$ value of which the triple is exactly the same as the distance between the pentagonal planes opposite in the small cage.

${ }^{35}$ F. Dubois and T. F. Fässler, J. Am. Chem. Soc. 127, 3264 (2005).

${ }^{36}$ F. D. Murnaghan, Proc. Natl. Acad. Sci. U.S.A. 30, 244 (1944).

${ }^{37}$ J. Dong and O. F. Sankey, J. Phys.: Condens. Mater. 11(32), 6129 (1999)

${ }^{38}$ C. S. Menoni, J. Z. Hu, and I. L. Spain, Phys. Rev. B 34, 362 (1986).

${ }^{39}$ M. I. McMahon, R. J. Nelmes, N. G. Wright, and D. R. Allan, Phys. Rev. B 50, 739 (1994).

${ }^{40}$ Y. Guyot, L. Grosvalet, B. Champagnon, E. Reny, C. Cros, and M. Pouchard, Phys. Rev. B 60, 14507 (1999).

${ }^{41}$ B. A. Weinstein and G. J. Piermarini, Phys. Rev. B 12, 1172 (1975). 